\title{
Multiple Periodic Solutions of Delayed Predator-Prey Systems with Type IV Functional Responses on Time Scales
}

\author{
Shengbin $\mathrm{Yu}^{1}{ }^{1}$ Haihui $\mathrm{Wu}^{1}{ }^{1}$ and Jiangbin $\mathrm{Chen}^{2}$ \\ ${ }^{1}$ Sunshine College, Fuzhou University, Fujian, Fuzhou 350015, China \\ ${ }^{2}$ Zhicheng College, Fuzhou University, Fujian, Fuzhou 350002, China \\ Correspondence should be addressed to Shengbin Yu, yushengbin.8@163.com
}

Received 11 August 2011; Accepted 26 December 2011

Academic Editor: Cengiz Çinar

Copyright (c) 2012 Shengbin Yu et al. This is an open access article distributed under the Creative Commons Attribution License, which permits unrestricted use, distribution, and reproduction in any medium, provided the original work is properly cited.

With the help of a continuation theorem based on Gaines and Mawhin's coincidence degree, easily verifiable criteria are established for the existence of multiple positive periodic solutions of delayed predator-prey systems with type IV functional responses on time scales. Our results not only unify the existing ones but also widen the range of applications.

\section{Introduction}

As was pointed out by Berryman [1], the dynamic relationship between predators and their prey has long been and will continue to be one of the dominant themes in both ecology and mathematical ecology due to its universal existence and importance. At first sight, these problems may appear to be simple mathematically. However, in fact, they are often very challenging and complicated. Also, Zhen and Ma [2] argued that the environmental fluctuation is important in an ecosystem, and more realistic models require the inclusion of the effect of environmental changes, especially environmental parameters which are time dependent and periodically changing (e.g., seasonal changes, food supplies, etc.). Hence, just as pointed out by Freedman and $\mathrm{Wu}$ [3] and Kuang [4], it would be of great interest and importance to study the existence of periodic solutions for systems with periodic delay. Much progress has been made in this direction (see, e.g., [5-8] and the references cited therein).

In 1959, in order to describe behavior of different kinds of species, Holling [9] proposed three types of functional response functions. However, some authors [10] have also described a type IV functional response that is humped and that declines at high prey densities. This decline may occur due to prey group defense or prey toxicity. Recently, Chen 
[11] has studied the following periodic predator-prey system with a type IV functional response:

$$
\begin{aligned}
& \dot{x}(t)=x(t)\left[b_{1}(t)-a_{1}(t) x\left(t-\tau_{1}(t)\right)-\frac{c(t) y(t-\sigma(t))}{\left(x^{2}(t) / n\right)+x(t)+a}\right], \\
& \dot{y}(t)=y(t)\left[-b_{2}(t)+\frac{a_{2}(t) x\left(t-\tau_{2}(t)\right)}{\left(x^{2}\left(t-\tau_{2}(t)\right) / n\right)+x\left(t-\tau_{2}(t)\right)+a}\right],
\end{aligned}
$$

where $c, \sigma, a_{j}, b_{j}$, and $\tau_{j}(j=1,2)$ are continuous $\omega$-periodic functions with $c(t) \geq$ $0, \sigma(t) \geq 0, a_{j}(t) \geq 0$, and $\tau_{j} \geq 0, \int_{0}^{\omega} c(t) d t>0$, and $\int_{0}^{\omega} b_{j}(t) d t>0, n$ and $a$ are positive constants. The growth functions $b_{j}$ may change sign, since the environment fluctuates randomly. Under bad conditions, $b_{j}$ may be negative.

Considering that discrete time models governed by difference equations are more appropriate than continuous ones when the populations have nonoverlapping generations, Zhang et al. [12] studied the following discrete time predator-prey system:

$$
\begin{gathered}
x(k+1)=x(k) \exp \left[b_{1}(k)-a_{1}(k) x\left(k-\tau_{1}(k)\right)-\frac{c(k) y(k-\sigma(k))}{\left(x^{2}(k) / n\right)+x(k)+a}\right], \\
y(k+1)=y(k) \exp \left[-b_{2}(k)+\frac{a_{2}(k) x\left(k-\tau_{2}(k)\right)}{\left(x^{2}\left(k-\tau_{2}(k)\right) / n\right)+x\left(k-\tau_{2}(k)\right)+a}\right]
\end{gathered}
$$

where, for $i=1,2, b_{i}: \mathbb{Z} \rightarrow \mathbb{R}, c, a_{i}: \mathbb{Z} \rightarrow \mathbb{R}^{+}, \tau_{i}, \sigma: \mathbb{Z} \rightarrow \mathbb{Z}^{+}$are all $\omega$-periodic.

On the other hand, recently, Bohner et al. [13] pointed out that it is unnecessary to explore the existence of periodic solutions of some continuous and discrete population models in separate ways. One can unify such studies in the sense of dynamic equation on general time scales. The theory of calculus on time scales, which has recently received a lot of attention, was initiated by Hilger in his Ph.D. Thesis in 1988 [14] in order to unify continuous and discrete analysis. Although there has been much research activity concerning the oscillation (nonoscillation) of solutions and periodic solution of differential equation on time scales (or measure chains) (see, e.g., [15-29]), there are few results dealing with multiple periodic solutions of predator-prey systems with time delay.

Motivated by the above work, we consider the following system on time scales $\mathbb{T}$ :

$$
\begin{gathered}
x^{\Delta}(t)=b_{1}(t)-a_{1}(t) \exp \left\{x\left(t-\tau_{1}(t)\right)\right\}-\frac{c(t) \exp \{y(t-\tau(t))\}}{\exp \{2 x(t)\} / n+\exp \{x(t)\}+a}, \\
y^{\Delta}(t)=-b_{2}(t)+\frac{a_{2}(t) \exp \left\{x\left(t-\tau_{2}(t)\right)\right\}}{\exp \left\{2 x\left(t-\tau_{2}(t)\right)\right\} / n+\exp \left\{x\left(t-\tau_{2}(t)\right)\right\}+a},
\end{gathered}
$$

where, for $i=1,2, b_{i}: \mathbb{T} \rightarrow \mathbb{R}, c, a_{i}, \tau_{i}, \tau: \mathbb{T} \rightarrow \mathbb{R}^{+}$are $\omega$-periodic functions. $n$ and $a$ are positive constants.

The main purpose of this paper is to derive a set of easily verifiable sufficient conditions for the existence of multiple positive periodic solutions of (1.3). The method used here will be the coincidence degree theory developed by Gaines and Mawhin [30].

In (1.3), set $z_{1}(t)=\exp \{x(t)\}, z_{2}(t)=\exp \{y(t)\}$. If $\mathbb{T}=\mathbb{R}$, then (1.3) reduces to (1.1). Also, if $\mathbb{T}=\mathbb{Z}$, then (1.3) becomes (1.2). Thus, our results also show that it is unnecessary to 
explore the existence of periodic solutions of continuous and discrete population models in separate ways. One can unify such studies in the sense of dynamic equations on time scales.

The paper is arranged as follows. In Section 2, we present some preliminary results such as the calculus on time scales and the continuation theorem in coincidence degree theory. In Section 3, we prove our main result.

\section{Preliminaries}

In this section, we give a short introduction to the time scales calculus and recall the continuation theorem from coincidence degree theory.

First, let us present some foundational definitions and results from the calculus on time scales, for proofs and further explanation and results, we refer to the paper by Hilger [14].

Definition 2.1. A time scale is an arbitrary nonempty closed subset $\mathbb{T}$ of the real numbers $\mathbb{R}$. The set $\mathbb{T}$ inherits the standard topology of $\mathbb{R}$.

Definition 2.2. For $t \in \mathbb{T}$, one defines the forward jump operator $\sigma: \mathbb{T} \rightarrow \mathbb{T}$ by

$$
\sigma(t):=\inf \{s \in \mathbb{T}: s>t\}
$$

while the backward jump operator $\rho: \mathbb{T} \rightarrow \mathbb{T}$ is defined by

$$
\rho(t):=\sup \{s \in \mathbb{T}: s<t\}
$$

In this definition we put $\inf \emptyset=\sup \mathbb{T}$ (i.e., $\sigma(t)=t$ if $\mathbb{T}$ has a maximum $t$ ) and sup $\emptyset=\inf \mathbb{T}$ (i.e., $\rho(t)=t$ if $\mathbb{T}$ has a minimum $t$ ), where $\emptyset$ denotes the empty set. If $\sigma(t)>t$, we say that $t$ is right-scattered, while if $\rho(t)<t$ we say that $t$ is left-scattered. Points that are right-scattered and left-scattered at the same time are called isolated. Also, if $t<\sup \mathbb{T}$ and $\sigma(t)=t$, then $t$ is called right-dense, and if $t>\inf \mathbb{T}$ and $\rho(t)=t$, then $t$ is called left-dense. Points that are right-dense and left-dense at the same time are called dense.

Definition 2.3. A function $f: \mathbb{T} \rightarrow \mathbb{R}$ is said to be rd-continuous if it is continuous at rightdense points in $\mathbb{T}$ and its left-sided limits exist (finite) at left-dense points in $\mathbb{T}$. The set of rd-continuous functions is denoted by $C_{\mathrm{rd}}=C_{\mathrm{rd}}(\mathbb{T})=C_{\mathrm{rd}}(\mathbb{T}, \mathbb{R})$.

Definition 2.4. Suppose $f: \mathbb{T} \rightarrow \mathbb{R}$ is a function, and let $t \in \mathbb{T}$. Then one defines $f^{\Delta}(t)$, the delta-derivative of $f$ at $t$, to be the number (provided it exists) with the property that, given any $\varepsilon>0$, there is a neighborhood $U$ of $t$ (i.e., $U=(t-\delta, t+\delta) \cap \mathbb{T}$ ) for some $\delta>0$ such that

$$
\left|[f(\sigma(t))-f(s)]-f^{\Delta}(t)[\sigma(t)-s]\right| \leq \varepsilon|\sigma(t)-s| \quad \forall s \in U
$$

Thus, $f$ is said to be delta-differentiable if its delta-derivative exists. The set of functions $f$ : $\mathbb{T} \rightarrow \mathbb{R}$ that are delta-differentiable and whose delta-derivative are rd-continuous functions is denoted by $C_{\mathrm{rd}}^{1}=C_{\mathrm{rd}}^{1}(\mathbb{T})=C_{\mathrm{rd}}^{1}(\mathbb{T}, \mathbb{R})$. 
Definition 2.5. A function $F: \mathbb{T} \rightarrow \mathbb{R}$ is called a delta-antiderivative of $f: \mathbb{T} \rightarrow \mathbb{R}$ provided $F^{\Delta}=f(t)$, for all $t \in \mathbb{T}$. Then, one writes

$$
\int_{r}^{s} f(t) \Delta t:=F(s)-F(r) \quad \forall s, r \in \mathbb{T} .
$$

Definition 2.6. One says that a time scale $\mathbb{T}$ is $\omega$-periodic, if $t \in \mathbb{T}$ implies $t+\omega \in \mathbb{T}$.

Lemma 2.7. Every rd-continuous function has an antiderivative.

Lemma 2.8. If $a, b \in \mathbb{T}, \alpha, \beta \in \mathbb{R}$, and $f, g \in C_{r d}(\mathbb{T})$, then

(i) $\int_{a}^{b}[\alpha f(t)+\beta g(t)] \Delta t=\alpha \int_{a}^{b} f(t) \Delta t+\beta \int_{a}^{b} g(t) \Delta t$;

(ii) if $f(t) \geq 0$ for all $a \leq t<b$, then $\int_{a}^{b} f(t) \Delta t \geq 0$;

(iii) if $|f(t)| \leq g(t)$ on $[a, b):=\{t \in \mathbb{T}: a \leq t<b\}$, then $\left|\int_{a}^{b} f(t) \Delta t\right| \leq \int_{a}^{b} g(t) \Delta t$.

Let

For convenience, one now introduces some notations to be used throughout this paper.

$$
\begin{gathered}
\mathcal{\kappa}=\min \{[0, \infty) \cap \mathbb{T}\}, \quad I_{\omega}=[\kappa, \kappa+\omega] \cap \mathbb{T}, \quad g^{u}=\sup _{t \in \mathbb{T}} g(t), \\
g^{l}=\inf _{t \in \mathbb{T}} g(t), \quad \bar{g}=\frac{1}{\omega} \int_{I_{\omega}} g(t) \Delta t=\int_{\kappa}^{\kappa+\omega} g(t) \Delta t,
\end{gathered}
$$

where $g \in C_{\text {rd }}(\mathbb{T})$ is an $\omega$-periodic real function.

In order to obtain the existence of positive periodic solutions of (1.3), for the reader's convenience, we will summarize in the following a few concepts and results from [30] that will be basic for this paper.

Let $X, Z$ be normed vector spaces, $L$ : Dom $L \subset X \rightarrow Z$ a linear mapping, and $N: X \rightarrow Z$ a continuous mapping. The mapping $L$ will be called a Fredholm mapping of index zero if $\operatorname{dim} \operatorname{Ker} L=$ Codim $\operatorname{Im} L<+\infty$ and $\operatorname{Im} L$ is closed in $Z$. If $L$ is a Fredholm mapping of index zero, there exist continuous projectors $P: X \rightarrow X$ and $Q: Z \rightarrow Z$ such that $\operatorname{Im} P=\operatorname{Ker} L, \operatorname{Im} L=\operatorname{Ker} Q=\operatorname{Im}(I-Q)$. It follows that $L \mid \operatorname{Dom} L \cap \operatorname{Ker} P$ : $(I-P) X \rightarrow \operatorname{Im} L$ is invertible. We denote the inverse of that map by $K_{P}$. Let $\Omega$ be an open bounded subset of $X$; the mapping $N$ will be called $L$-compact on $\bar{\Omega}$ if $Q N(\bar{\Omega})$ is bounded and $K_{P}(I-Q) N: \bar{\Omega} \rightarrow X$ is compact. Since $\operatorname{Im} Q$ is isomorphic to Ker $L$, there exists an isomorphism $J: \operatorname{Im} Q \rightarrow \operatorname{Ker} L$.

Lemma 2.9 (continuation theorem). Let $L$ be a Fredholm mapping of index zero, and let $N$ be L-compact on $\bar{\Omega}$. Suppose

(a) for each $\lambda \in(0,1)$, every solution $x$ of $L x=\lambda N x$ is such that $x \notin \partial \Omega$;

(b) $Q N x \neq 0$ for each $x \in \partial \Omega \cap$ Ker $L$ and

$$
\operatorname{deg}\{J Q N, \Omega \cap \operatorname{Ker} L, 0\} \neq 0 .
$$

Then the equation $L x=N x$ has at least one solution lying in Dom $L \cap \bar{\Omega}$. 
Now, we give a lemma which will be useful in our following proof. The proofs of the lemmas can be found in [13].

Lemma 2.10. Let $t_{1}, t_{2} \in I_{\omega}$ and $t \in \mathbb{T}$. If $f: \mathbb{T} \rightarrow \mathbb{R}$ is $\omega$-periodic, then

$$
f(t) \leq f\left(t_{1}\right)+\int_{I_{\omega}}\left|f^{\Delta}\right| \Delta s, \quad f(t) \geq f\left(t_{2}\right)-\int_{I_{\omega}}\left|f^{\Delta}\right| \Delta s .
$$

\section{Existence of Periodic Solutions}

The goal of this section is to establish sufficient conditions on the existence of periodic solution for system (1.3), where, for $i=1,2, b_{i}, c, a_{i}, \tau_{i}, \tau$ are rd-continuous functions. Firstly, we always assume that

$$
\overline{a_{2}}>\overline{b_{2}}\left(1+2 \sqrt{\frac{a}{n}}\right) \exp \left\{\left(\overline{\left|b_{1}\right|}+\overline{b_{1}}\right) \omega\right\}
$$

For further convenience, we define the following six positive numbers:

$$
\begin{aligned}
& l_{ \pm}=\frac{n\left[\overline{a_{2}} \exp \left\{\left(\overline{\left|\overline{b_{1}}\right|}+\overline{b_{1}}\right) \omega\right\}-\overline{b_{2}}\right] \pm \sqrt{n^{2}\left[\overline{a_{2}} \exp \left\{\left(\overline{\left|\overline{b_{1}}\right|}+\overline{b_{1}}\right) \omega\right\}-\overline{b_{2}}\right]^{2}-4 n a{\overline{b_{2}}}^{2}}}{2 \overline{b_{2}}}, \\
& u_{ \pm}=\frac{n\left[\overline{a_{2}}-\overline{b_{2}} \exp \left\{\left(\overline{\left|\overline{b_{1}}\right|}+\overline{b_{1}}\right) \omega\right\}\right] \pm \sqrt{n^{2}\left[\overline{a_{2}}-\overline{b_{2}} \exp \left\{\left(\overline{\left|\overline{b_{1}}\right|}+\overline{b_{1}}\right) \omega\right\}\right]^{2}-4 n a{\overline{b_{2}}}^{2} \exp \left\{2\left(\overline{\left|\overline{b_{1}}\right|}+\overline{b_{1}}\right) \omega\right\}}}{2 \overline{b_{2}} \exp \left\{\left(\overline{b_{1} \mid}+\overline{b_{1}}\right) \omega\right\}}, \\
& v_{ \pm}=\frac{n\left(\overline{a_{2}}-\overline{b_{2}}\right) \pm \sqrt{n^{2}\left(\overline{a_{2}}-\overline{b_{2}}\right)^{2}-4 n a{\overline{b_{2}}}^{2}}}{2 \overline{b_{2}}} .
\end{aligned}
$$

It is easy to show that

$$
l_{-}<v_{-}<u_{-}<u_{+}<v_{+}<l_{+}
$$

We now come to the main result of this paper.

Theorem 3.1. In addition to $\left(H_{1}\right)$, assume further that

$$
\overline{a_{1}} l_{+} \exp \left\{\left(\overline{\left|b_{1}\right|}+\overline{b_{1}}\right) \omega\right\}<\overline{b_{1}}
$$

holds and the system (1.3) has at least two w-periodic solutions. 
Proof. In order to apply Lemma 2.9 (continuation theorem) to (1.3), we first define

$$
\begin{gathered}
X=Z=\left\{(x, y)^{T} \mid x, y \in C_{\mathrm{rd}}, x(t+\omega)=x(t), y(t+\omega)=y(t)\right\}, \\
\left\|(x, y)^{T}\right\|=\max _{t \in T_{\omega}}|x(t)|+\max _{t \in T_{\omega}}|y(t)|
\end{gathered}
$$

for any $(x, y) \in X$ (or $Z$ ). Then $X, Z$ are both Banach spaces when they are endowed with the above norm $\|\cdot\|$.

For $\left[\begin{array}{l}x \\ y\end{array}\right] \in X$, we define

$$
\begin{aligned}
N\left[\begin{array}{l}
x \\
y
\end{array}\right]=\left[\begin{array}{l}
N_{1}(t) \\
N_{2}(t)
\end{array}\right]= & {\left[\begin{array}{c}
b_{1}(t)-a_{1}(t) \exp \left\{x\left(t-\tau_{1}(t)\right)\right\}-\frac{c(t) \exp \{y(t-\tau(t))\}}{\exp \{2 x(t)\} / n+\exp \{x(t)\}+a} \\
-b_{2}(t)+\frac{a_{2}(t) \exp \left\{x\left(t-\tau_{2}(t)\right)\right\}}{\exp \left\{2 x\left(t-\tau_{2}(t)\right)\right\} / n+\exp \left\{x\left(t-\tau_{2}(t)\right)\right\}+a}
\end{array}\right], } \\
L\left[\begin{array}{l}
x \\
y
\end{array}\right] & =\left[\begin{array}{l}
x^{\Delta} \\
y^{\Delta}
\end{array}\right], \quad P\left[\begin{array}{l}
x \\
y
\end{array}\right]=Q\left[\begin{array}{l}
x \\
y
\end{array}\right]=\left[\begin{array}{l}
\frac{1}{\omega} \int_{I_{\omega}} x(t) \Delta t \\
\frac{1}{\omega} \int_{I_{\omega}} y(t) \Delta t
\end{array}\right]=\left[\begin{array}{l}
\bar{x} \\
\bar{y}
\end{array}\right] .
\end{aligned}
$$

Then, it follows that

$$
\begin{gathered}
\operatorname{Ker} L=\left\{(x, y)^{T} \in X \mid(x, y)^{T}=\left(h_{1}, h_{2}\right)^{T} \in \mathbb{R}^{2} \text { for } t \in \mathbb{T}\right\}, \\
\operatorname{Im} L=\left\{(x, y)^{T} \in X \mid \int_{I_{\omega}} x(t) \Delta t=0, \int_{I_{\omega}} y(t) \Delta t t \in \mathbb{T}\right\} \quad \text { is closed in } Z,
\end{gathered}
$$

$\operatorname{dim} \operatorname{Ker} L=2=$ Codim $\operatorname{Im} L$,

and $P, Q$ are continuous projectors such that

$$
\operatorname{Im} P=\operatorname{Ker} L, \quad \text { Ker } Q=\operatorname{Im} L=\operatorname{Im}(I-Q) .
$$

Therefore, $L$ is a Fredholm mapping of index zero. Furthermore, the generalized inverse (to $L$ ) $K_{P}: \operatorname{Im} L \rightarrow \operatorname{Ker} P \cap$ Dom $L$ reads

$$
K_{P}\left[\begin{array}{l}
x \\
y
\end{array}\right]=\left[\begin{array}{l}
\int_{\kappa}^{t} x(s) \Delta s-\frac{1}{\omega} \int_{\kappa}^{\kappa+\omega} \int_{\kappa}^{t} x(s) \Delta s \Delta t \\
\int_{\kappa}^{t} y(s) \Delta s-\frac{1}{\omega} \int_{\kappa}^{\kappa+\omega} \int_{\kappa}^{t} y(s) \Delta s \Delta t
\end{array}\right] .
$$


Thus,

$$
\begin{gathered}
Q N\left[\begin{array}{l}
x \\
y
\end{array}\right]=\left[\begin{array}{c}
\frac{1}{\omega} \int_{\kappa}^{\kappa+\omega}\left[b_{1}(s)-a_{1}(s) \exp \left\{x\left(t-\tau_{1}(s)\right)\right\}-\frac{c(s) \exp \{y(s-\tau(s))\}}{\exp \{2 x(s)\} / n+\exp \{x(s)\}+a}\right] \Delta s \\
\frac{1}{\omega} \int_{\kappa}^{\kappa+\omega}\left[-b_{2}(s)+\frac{a_{2}(s) \exp \left\{x\left(t-\tau_{2}(s)\right)\right\}}{\exp \left\{2 x\left(s-\tau_{2}(s)\right)\right\} / n+\exp \left\{x\left(s-\tau_{2}(s)\right)\right\}+a}\right] \Delta s
\end{array}\right], \\
K_{P}(I-Q) N\left[\begin{array}{l}
x \\
y
\end{array}\right]=\left[\begin{array}{l}
\int_{\kappa}^{t} N_{1}(s) \Delta s-\frac{1}{\omega} \int_{\kappa}^{\kappa+\omega} \int_{\kappa}^{t} N_{1}(s) \Delta s \Delta t-\left(t-\kappa-\frac{1}{\omega} \int_{\kappa}^{\kappa+\omega}(t-\kappa) \Delta t\right) \overline{N_{1}} \\
\int_{\kappa}^{t} N_{2}(s) \Delta s-\frac{1}{\omega} \int_{\kappa}^{\kappa+\omega} \int_{\kappa}^{t} N_{2}(s) \Delta s \Delta t-\left(t-\kappa-\frac{1}{\omega} \int_{\kappa}^{\kappa+\omega}(t-\kappa) \Delta t\right) \overline{N_{2}}
\end{array}\right] .
\end{gathered}
$$

Obviously, $Q N$ and $K_{P}(I-Q) N$ are continuous. It is not difficult to show that $\overline{K_{P}(I-Q) N(\bar{\Omega})}$ is compact for any open bounded set $\Omega \subset Z$ by using the Arzela-Ascoli theorem. Moreover, $Q N(\bar{\Omega})$ is clearly bounded. Thus, $N$ is $L$-compact on $\bar{\Omega}$ with any open bounded set $\Omega \subset Z$.

Now we reach the position to search for an appropriate open bounded subset $\Omega$ for the application of the continuation theorem (Lemma 2.9). Corresponding to the operator equation $L x=\lambda N x, L y=\lambda N y, \lambda \in(0,1)$, we have

$$
\begin{gathered}
x^{\Delta}(t)=\lambda\left[b_{1}(t)-a_{1}(t) \exp \left\{x\left(t-\tau_{1}(t)\right)\right\}-\frac{c(t) \exp \{y(t-\tau(t))\}}{\exp \{2 x(t)\} / n+\exp \{x(t)\}+a}\right], \\
y^{\Delta}(t)=\lambda\left[-b_{2}(t)+\frac{a_{2}(t) \exp \left\{x\left(t-\tau_{2}(t)\right)\right\}}{\exp \left\{2 x\left(t-\tau_{2}(t)\right)\right\} / n+\exp \left\{x\left(t-\tau_{2}(t)\right)\right\}+a}\right] .
\end{gathered}
$$

Suppose that $(x(t), y(t))^{T} \in X$ is a solution of system (3.9) for a certain $\mathcal{\lambda} \in(0,1)$. Integrating (3.2) over the set $I_{\omega}$, we obtain

$$
\begin{gathered}
\overline{b_{1}} \omega=\int_{I_{\omega}}\left[a_{1}(t) \exp \left\{x\left(t-\tau_{1}(t)\right)\right\}+\frac{c(t) \exp \{y(t-\tau(t))\}}{\exp \{2 x(t)\} / n+\exp \{x(t)\}+a}\right] \Delta t, \\
\overline{b_{2}} \omega=\int_{I_{\omega}}\left[\frac{a_{2}(t) \exp \left\{x\left(t-\tau_{2}(t)\right)\right\}}{\exp \left\{2 x\left(t-\tau_{2}(t)\right)\right\} / n+\exp \left\{x\left(t-\tau_{2}(t)\right)\right\}+a}\right] \Delta t .
\end{gathered}
$$


It follows from (3.9)-(3.11) that

$$
\begin{aligned}
\int_{I_{\omega}}\left|x^{\Delta}(t)\right| \Delta t & <\int_{I_{\omega}}\left|b_{1}(t)\right| \Delta t+\int_{I_{\omega}}\left[a_{1}(t) \exp \left\{x\left(t-\tau_{1}(t)\right)\right\}+\frac{c(t) \exp \{y(t-\tau(t))\}}{\exp \{2 x(t)\} / n+\exp \{x(t)\}+a}\right] \Delta t \\
& =\left(\overline{\left|b_{1}\right|}+\overline{b_{1}}\right) \omega, \\
\int_{I_{\omega}}\left|y^{\Delta}(t)\right| \Delta t & <\int_{I_{\omega}}\left|b_{2}(t)\right| \Delta t+\int_{I_{\omega}}\left[\frac{a_{2}(t) \exp \left\{x\left(t-\tau_{2}(t)\right)\right\}}{\exp \left\{2 x\left(t-\tau_{2}(t)\right)\right\} / n+\exp \left\{x\left(t-\tau_{2}(t)\right)\right\}+a}\right] \Delta t \\
& =\left(\overline{\left|b_{2}\right|}+\overline{b_{2}}\right) \omega .
\end{aligned}
$$

Note that $(x(t), y(t))^{T} \in X$, then there exist $\xi_{i}, \eta_{i} \in I_{\omega}, i=1,2$, such that

$$
\begin{aligned}
& x\left(\xi_{1}\right)=\min _{t \in I_{\omega}} x(t), \quad x\left(\eta_{1}\right)=\max _{t \in I_{\omega}} x(t), \\
& y\left(\xi_{2}\right)=\min _{t \in I_{\omega}} y(t), \quad y\left(\eta_{2}\right)=\max _{t \in I_{\omega}} y(t) .
\end{aligned}
$$

Then, By (3.11) and (3.14), we have

$$
\overline{b_{2}} \omega \leq \int_{I_{\omega}}\left[\frac{a_{2}(t) \exp \left\{x\left(\eta_{1}\right)\right\}}{\exp \left\{2 x\left(\xi_{1}\right)\right\} / n+\exp \left\{x\left(\xi_{1}\right)\right\}+a}\right] \Delta t=\frac{\overline{a_{2}} \omega \exp \left\{x\left(\eta_{1}\right)\right\}}{\exp \left\{2 x\left(\xi_{1}\right)\right\} / n+\exp \left\{x\left(\xi_{1}\right)\right\}+a},
$$

that is,

$$
x\left(\eta_{1}\right) \geq \ln \left[\frac{\overline{b_{2}}\left(\exp \left\{2 x\left(\xi_{1}\right)\right\} / n+\exp \left\{x\left(\xi_{1}\right)\right\}+a\right)}{\overline{a_{2}}}\right] .
$$

According to (3.12), (3.16), and Lemma 2.10, we derive

$$
x(t) \geq x\left(\eta_{1}\right)-\int_{I_{\omega}}\left|x^{\Delta}\right| \Delta t>\ln \left[\frac{\overline{b_{2}}\left(\exp \left\{2 x\left(\xi_{1}\right)\right\} / n+\exp \left\{x\left(\xi_{1}\right)\right\}+a\right)}{\overline{a_{2}}}\right]-\left(\overline{\left|b_{1}\right|}+\overline{b_{1}}\right) \omega .
$$

In particular, we have

$$
x\left(\xi_{1}\right)>\ln \left[\frac{\overline{b_{2}}\left(\exp \left\{2 x\left(\xi_{1}\right)\right\} / n+\exp \left\{x\left(\xi_{1}\right)\right\}+a\right)}{\overline{a_{2}}}\right]-\left(\overline{\left|b_{1}\right|}+\overline{b_{1}}\right) \omega
$$

or

$$
\frac{\overline{b_{2}}}{n} \exp \left\{2 x\left(\xi_{1}\right)\right\}-\left(\overline{a_{2}} \exp \left\{\overline{\left|b_{1}\right|}+\overline{b_{1}}\right\}-\overline{b_{2}}\right) \exp \left\{x\left(\xi_{1}\right)\right\}+\overline{b_{2}} a<0
$$


Discrete Dynamics in Nature and Society

According to $\left(H_{1}\right)$, we have

$$
\ln l_{-}<x\left(\xi_{1}\right)<\ln l_{+} .
$$

Similarly, we also can obtain

$$
x\left(\eta_{1}\right)<\ln u_{-} \quad \text { or } \quad x\left(\eta_{1}\right)<\ln u_{+} .
$$

From (3.12) and (3.20) and Lemma 2.10, one has

$$
x(t) \leq x\left(\xi_{1}\right)+\int_{I_{\omega}}\left|x^{\Delta}\right| \Delta t<\ln l_{+}+\left(\overline{\left|b_{1}\right|}+\overline{b_{1}}\right) \omega \stackrel{\text { def }}{=} M_{1} .
$$

This, combined with (3.10) and (3.14), gives

$$
\begin{gathered}
\overline{b_{1}} \omega \geq \frac{\bar{c} \omega \exp \left\{y\left(\xi_{2}\right)\right\}}{\exp \left\{2 M_{1}\right\} / n+\exp \left\{M_{1}\right\}+a}, \\
\overline{b_{1}} \omega \leq \overline{a_{1}} \omega \exp \left\{M_{1}\right\}+\frac{\bar{c} \omega \exp \left\{y\left(\eta_{2}\right)\right\}}{a}
\end{gathered}
$$

It follows from (3.23) that

$$
y\left(\xi_{2}\right) \leq \ln \left\{\frac{\overline{b_{1}}\left[\exp \left\{2 M_{1}\right\} / n+\exp \left\{M_{1}\right\}+a\right]}{\overline{c_{1}}}\right\}
$$

This, together with (3.13) and Lemma 2.10, yields

$$
y(t) \leq y\left(\xi_{2}\right)+\int_{I_{\omega}}\left|y^{\Delta}\right| \Delta t<\ln \left\{\frac{\overline{b_{1}}\left[\exp \left\{2 M_{1}\right\} / n+\exp \left\{M_{1}\right\}+a\right]}{\overline{c_{1}}}\right\}+\left(\overline{\left|b_{2}\right|}+\overline{b_{2}}\right) \omega \stackrel{\text { def }}{=} M_{2} .
$$

Moreover, because of $\left(\mathrm{H}_{2}\right)$, it follows from (3.24) that

$$
y\left(\eta_{2}\right) \geq \ln \frac{a\left[\overline{b_{1}}-\overline{a_{1}} l_{+} \exp \left\{\left(\overline{\left|b_{1}\right|}+\overline{b_{1}}\right) \omega\right\}\right]}{\bar{c}} .
$$

This, together with (3.13) and Lemma 2.10 again, yields

$$
y(t) \geq y\left(\eta_{2}\right)-\int_{I_{\omega}}\left|y^{\Delta}\right| \Delta t \geq \ln \frac{a\left[\overline{b_{1}}-\overline{a_{1}} l_{+} \exp \left\{\left(\overline{\left|b_{1}\right|}+\overline{b_{1}}\right) \omega\right\}\right]}{\bar{c}}-\left(\overline{\left|b_{2}\right|}+\overline{b_{2}}\right) \omega \stackrel{\text { def }}{=} m_{2} .
$$


It follows from (3.26) and (3.28) that

$$
\max _{t \in I_{\omega}}|y(t)|<\max \left\{\left|m_{2}\right|,\left|M_{2}\right|\right\} \stackrel{\text { def }}{=} M .
$$

Obviously, $\ln l_{ \pm}, \ln u_{ \pm}, M_{1}$, and $M$ are independent of $\lambda$.

Now, let us consider $Q N z_{1}$ with $z_{1}=(x, y)^{T} \in \mathbb{R}$. Note that

$$
Q N z_{1}=\left[\begin{array}{c}
\overline{b_{1}} \omega-\overline{a_{1}} \omega \exp \{x\}-\frac{\bar{c} \omega \exp \{y\}}{\exp \{2 x\} / n+\exp \{x\}+a} \\
-\overline{b_{2}} \omega+\frac{\overline{a_{2}} \omega \exp \{x\}}{\exp \{2 x\} / n+\exp \{x\}+a}
\end{array}\right] .
$$

By virtue of $\left(H_{1}\right)$ and $\left(H_{2}\right)$, we can show that $Q N z_{1}=0$ has two distinct solutions $z_{11}=$ $\left(\ln v_{-}, \ln \left(\left(\overline{b_{1}}-\overline{a_{1}} v_{-}\right)\left(v_{-}^{2} / n+v_{-}+a\right) / \bar{c}\right)\right)^{T}$ and $z_{12}=\left(\ln v_{+}, \ln \left(\left(\overline{b_{1}}-\overline{a_{1}} v_{+}\right)\left(v_{-}^{2} / n+v_{+}+a\right) / \bar{c}\right)\right)^{T}$. Choose $C>0$ such that

$$
C>\max \left\{\left|\frac{\left(\overline{b_{1}}-\overline{a_{1}} v_{-}\right)\left(v_{-}^{2} / n+v_{-}+a\right)}{\bar{c}}\right|,\left|\frac{\left(\overline{b_{1}}-\overline{a_{1}} v_{+}\right)\left(v_{-}^{2} / n+v_{+}+a\right)}{\bar{c}}\right|\right\} .
$$

Let

$$
\begin{aligned}
& \Omega_{1}=\left\{(x, y) \in X\left|x(t) \in\left(\ln l_{-}, \ln u_{-}\right), \max _{t \in I_{\omega}}\right| y(t) \mid<M+C\right\}, \\
& \Omega_{2}=\left\{(x, y) \in X \mid \min _{t \in I_{\omega}} x(t) \in\left(\ln l_{-}, \ln l_{+}\right), \max _{t \in I_{\omega}} x(t) \in\left(\ln u_{+}, M_{1}\right) \text { and } \max _{t \in I_{\omega}}|y(t)|<M+C\right\} .
\end{aligned}
$$

Then both $\Omega_{1}$ and $\Omega_{2}$ are bounded open subsets of X. It follows from (3.2) and (3.31) that $z_{11} \in \Omega_{1}$ and $z_{12} \in \Omega_{2}$. With the help of (3.2), (3.20)-(3.22), (3.29), and (3.31), it is easy to see that $\Omega_{1} \cap \Omega_{2}=\emptyset$, and $\Omega_{i}$ satisfy the requirement (a) in Lemma 2.9 for $i=1$, 2. Moreover, $Q N z \neq 0$ for $z \in \partial \Omega_{i} \cap \operatorname{Ker} L$. A direct calculation shows that

$$
\operatorname{deg}\left\{J Q N, \Omega_{i} \cap \operatorname{Ker} L, 0\right\}=(-1)^{i+1} \neq 0 .
$$

Here, $J$ can be the identity mapping since $\operatorname{Im} P=\operatorname{Ker} L$. So far, we have proved that $\Omega_{i}$ verifies all the requirements in Lemma 2.9. Hence (1.3) has at least two $\omega$-periodic solutions. This completes the proof.

Remark 3.2. In (1.3), set $z_{1}(t)=\exp \{x(t)\}, z_{2}(t)=\exp \{y(t)\}$. When $\mathbb{T}=\mathbb{R}$, then (1.3) reduces to (1.1). Also, if $\mathbb{T}=\mathbb{Z}$, then (1.3) becomes (1.2). Hence, our result unifies the main results of [11, Theorem 2.2] and [12, Theorem 2.1]. Moreover, our result will also be useful when 
$\mathbb{T}=h \mathbb{Z}=\{h k \mid k \in \mathbb{Z}\}$, where $h>0$ and $h \neq 1$; however, [11, Theorem 2.2] and [12, Theorem 2.1] are not applicable. In this case, we have

$$
\begin{aligned}
g^{u} & =\max _{0 \leq k \leq \omega / h-1} g(h k), \quad g^{l}=\min _{0 \leq k \leq \omega / h-1} g(h k), \\
\bar{g} & =\frac{1}{\omega} \sum_{k=0}^{\omega / h-1} g(h k) h, \quad f^{\Delta}(t)=\frac{f(t+h)-f(t)}{h},
\end{aligned}
$$

for every $\omega$-periodic sequence of positive real numbers, $g$ with $\omega>1$ and $t \in \mathbb{T}$.

Remark 3.3. According to the above proof, we also can obtain that Theorem 3.1 is true for the following general system:

$$
\begin{gathered}
x^{\Delta}(t)=b_{1}(t)-a_{1}(t) \exp \left(x\left(t-\tau_{1}(t)\right)\right)-\frac{c(t) \exp \{y(t-\tau(t))\}}{\exp \left\{2 x\left(t-\tau_{2}(t)\right)\right\} / n+\exp \left\{x\left(t-\tau_{2}(t)\right)\right\}+a}, \\
y^{\Delta}(t)=-b_{2}(t)+\frac{a_{2}(t) \exp \left\{x\left(t-\tau_{3}(t)\right)\right\}}{\exp \left\{2 x\left(t-\tau_{4}(t)\right)\right\} / n+\exp \left\{x\left(t-\tau_{4}(t)\right)\right\}+a} .
\end{gathered}
$$

Moreover, just the same as the authors in [11,12] point out, Theorem 3.1 will remain valid if some or all terms are replaced by terms with discrete time delays, distributed delays (finite or infinite), state-dependent delays, or deviating arguments. That is to say that time delays of any type or the deviating arguments can have no effect on the existence of positive periodic solutions.

\section{Acknowledgment}

The authors are deeply indebted to an anonymous referee for his excellent suggestions, which greatly improve the presentation of this paper.

\section{References}

[1] A. A. Berryman, "The origins and evolution of predator-prey theory," Ecology, vol. 73, no. 5, pp. 1530$1535,1992$.

[2] J. Zhen and Z. Ma, "Periodic solutions for delay differential equations model of plankton allelopathy," Computers \& Mathematics with Applications, vol. 44, no. 3-4, pp. 491-500, 2002.

[3] H. I. Freedman and J. H. Wu, "Periodic solutions of single-species models with periodic delay," SIAM Journal on Mathematical Analysis, vol. 23, no. 3, pp. 689-701, 1992.

[4] Y. Kuang, Delay Differential Equations with Applications in Population Dynamics, vol. 191 of Mathematics in Science and Engineering, Academic Press, Boston, Mass, USA, 1993.

[5] F. Chen, "Positive periodic solutions of neutral Lotka-Volterra system with feedback control," Applied Mathematics and Computation, vol. 162, no. 3, pp. 1279-1302, 2005.

[6] Y. Chen, "Periodic solutions of a delayed periodic logistic equation," Applied Mathematics Letters, vol. 16, no. 7, pp. 1047-1051, 2003.

[7] F. Chen and J. Shi, "Periodicity in a logistic type system with several delays," Computers \& Mathematics with Applications, vol. 48, no. 1-2, pp. 35-44, 2004.

[8] X. Chen, "Periodicity in a nonlinear discrete predator-prey system with state dependent delays," Nonlinear Analysis, vol. 8, no. 2, pp. 435-446, 2007. 
[9] C.S. Holling, "functional response of predator to prey density and its role in mimicry and population regulation," Memoirs of the Entomological Society of Canada, vol. 45, pp. 1-60, 1965.

[10] H. I. Freedman, Deterministic Mathematical Models in Population Ecology, vol. 57 of Monographs and Textbooks in Pure and Applied Mathematics, Marcel Dekker, New York, NY, USA, 1980.

[11] Y. Chen, "Multiple periodic solutions of delayed predator-prey systems with type IV functional responses," Nonlinear Analysis. Real World Applications, vol. 5, no. 1, pp. 45-53, 2004.

[12] W. Zhang, D. Zhu, and P. Bi, "Multiple positive periodic solutions of a delayed discrete predator-prey system with type IV functional responses," Applied Mathematics Letters, vol. 20, no. 10, pp. 1031-1038, 2007.

[13] M. Bohner, M. Fan, and J. Zhang, "Existence of periodic solutions in predator-prey and competition dynamic systems," Nonlinear Analysis. Real World Applications, vol. 7, no. 5, pp. 1193-1204, 2006.

[14] S. Hilger, "Analysis on measure chains-a unified approach to continuous and discrete calculus," Results in Mathematics, vol. 18, no. 1-2, pp. 18-56, 1990.

[15] M. Bohner and A. Peterson, Dynamic Equations on Time Scales: An Introduction with Applications, Birkhäuser, Boston, Mass, USA, 2001.

[16] S. Hilger, Advances in Dynamic Equations on Time Scales, Birkhäuser, Boston, Mass, USA, 2003.

[17] M. Bohner and P. W. Eloe, "Higher order dynamic equations on measure chains: Wronskians, disconjugacy, and interpolating families of functions," Journal of Mathematical Analysis and Applications, vol. 246, no. 2, pp. 639-656, 2000.

[18] B. G. Zhang and X. Deng, "Oscillation of delay differential equations on time scales," Mathematical and Computer Modelling, vol. 36, no. 11-13, pp. 1307-1318, 2002.

[19] L. Erbe and A. Peterson, "Oscillation criteria for second-order matrix dynamic equations on a time scale," Journal of Computational and Applied Mathematics, vol. 141, no. 1-2, pp. 169-185, 2002.

[20] L. Erbe and A. Peterson, "Green's functions and comparison theorems for differential equations on measure chains," Dynamics of Continuous, Discrete and Impulsive Systems, vol. 6, no. 1, pp. 121-137, 1999.

[21] L. Erbe and A. Peterson, "Positive solutions for a nonlinear differential equation on a measure chain," Mathematical and Computer Modelling, vol. 32, no. 5-6, pp. 571-585, 2000.

[22] W.-T. Li and H.-R. Sun, "Multiple positive solutions for nonlinear dynamical systems on a measure chain," Journal of Computational and Applied Mathematics, vol. 15, pp. 203-210, 2004.

[23] X.-L. Liu and W.-T. Li, "Periodic solutions for dynamic equations on time scales," Nonlinear Analysis, vol. 67, no. 5, pp. 1457-1463, 2007.

[24] L. Bi, M. Bohner, and M. Fan, "Periodic solutions of functional dynamic equations with infinite delay," Nonlinear Analysis, vol. 68, no. 5, pp. 1226-1245, 2008.

[25] M. Bohner, M. Fan, and J. Zhang, "Periodicity of scalar dynamic equations and applications to population models," Journal of Mathematical Analysis and Applications, vol. 330, no. 1, pp. 1-9, 2007.

[26] D. R. Anderson, "Multiple periodic solutions for a second-order problem on periodic time scales," Nonlinear Analysis, vol. 60, no. 1, pp. 101-115, 2005.

[27] X. Chen, "Periodicity in a nonlinear predator-prey system on time scales with state-dependent delays," Applied Mathematics and Computation, vol. 196, no. 1, pp. 118-128, 2008.

[28] M. Fazly and M. Hesaaraki, "Periodic solutions for predator-prey systems with BeddingtonDeAngelis functional response on time scales," Nonlinear Analysis. Real World Applications, vol. 9, no. 3, pp. 1224-1235, 2008.

[29] W. Zhang, P. Bi, and D. Zhu, "Periodicity in a ratio-dependent predator-prey system with stagestructured predator on time scales," Nonlinear Analysis. Real World Applications , vol. 9, no. 2, pp. 344-353, 2008.

[30] R. E. Gaines and J. L. Mawhin, Coincidence Degree, and Nonlinear Differential Equations, vol. 568 of Lecture Notes in Mathematics, Springer, Berlin, Germany, 1977. 


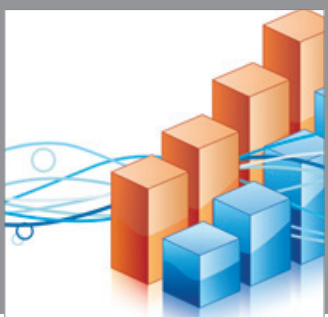

Advances in

Operations Research

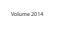

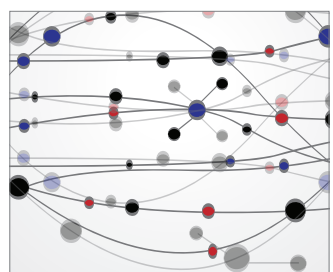

\section{The Scientific} World Journal
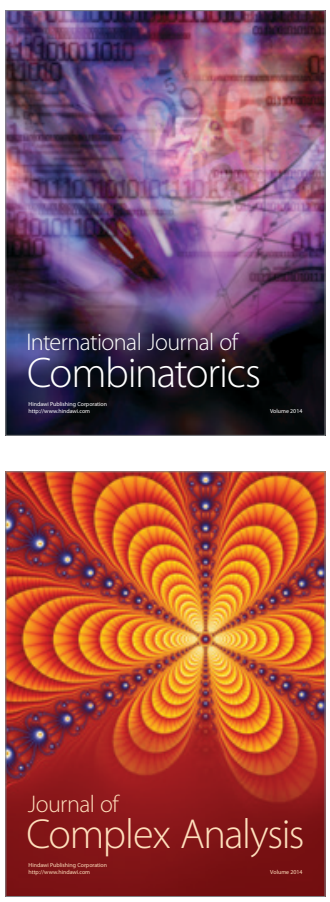

International Journal of

Mathematics and

Mathematical

Sciences
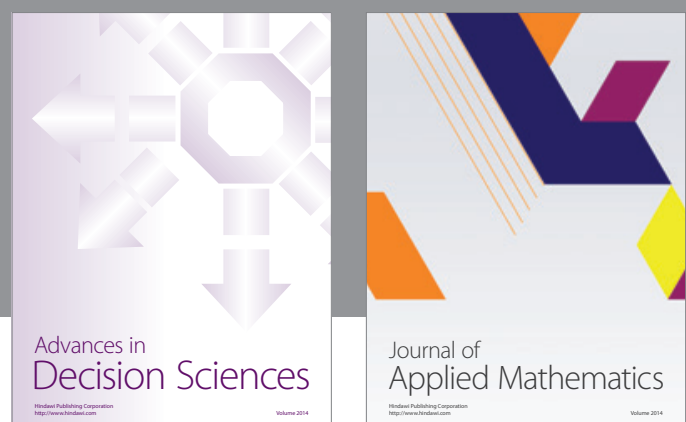

Journal of

Applied Mathematics
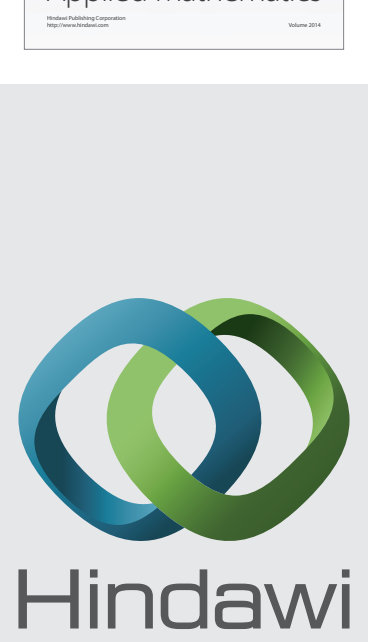

Submit your manuscripts at http://www.hindawi.com
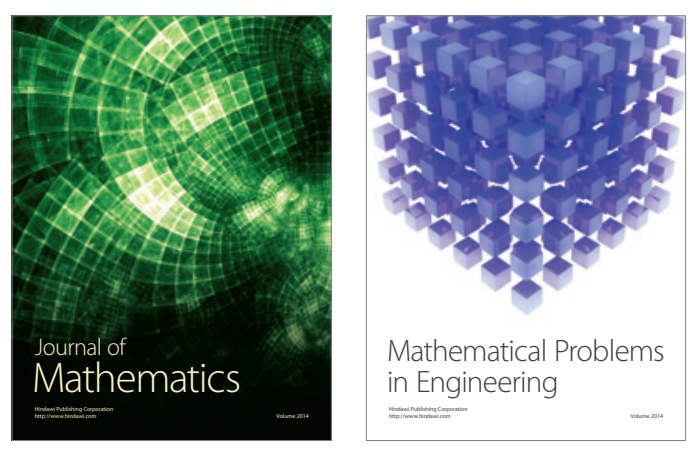

Mathematical Problems in Engineering
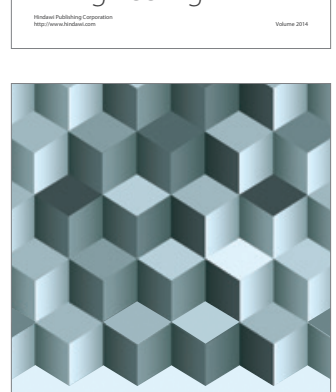

Journal of

Function Spaces
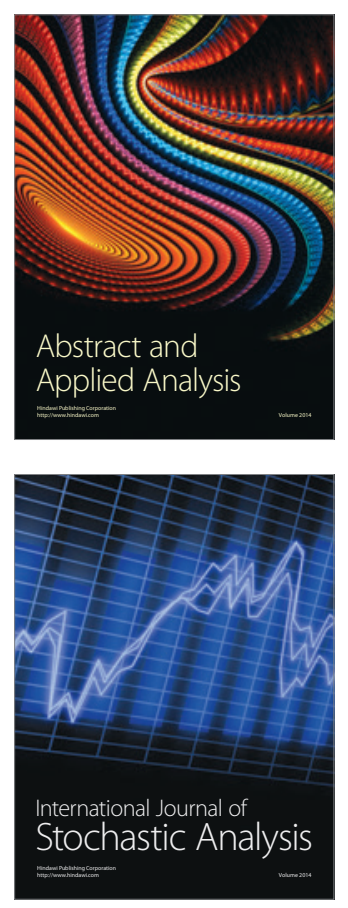

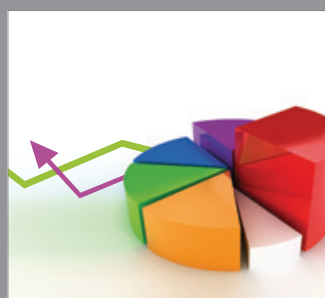

ournal of

Probability and Statistics

Promensencen
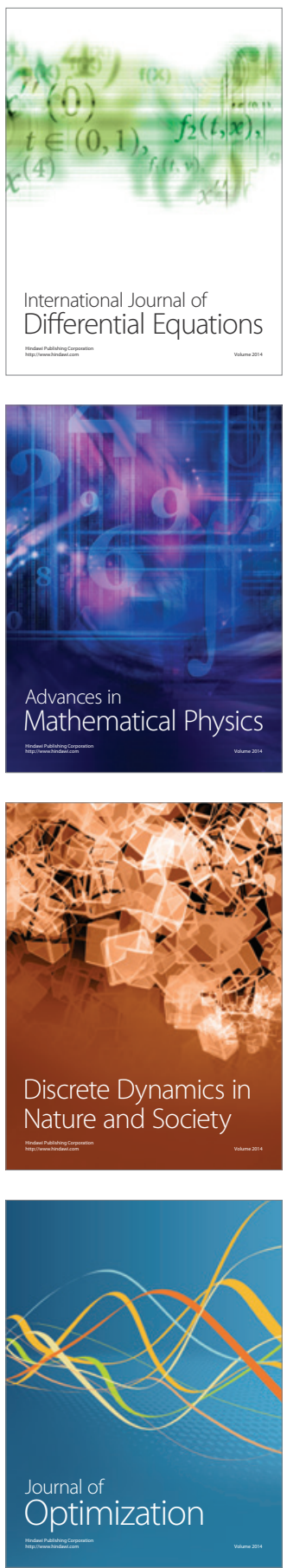